\title{
Massively ordered microstructures composed of magnetic nanoparticles
}

\author{
Myunghwan Byun, Jun Wang and Zhiqun $\operatorname{Lin}^{1}$ \\ Department of Materials Science and Engineering, Iowa State University, Ames, IA 50011, \\ USA \\ E-mail: zqlin@iastate.edu
}

Received 26 September 2008, in final form 31 October 2008

Published 11 June 2009

Online at stacks.iop.org/JPhysCM/21/264014

\begin{abstract}
Magnetic $\gamma-\mathrm{Fe}_{2} \mathrm{O}_{3}$ nanoparticle toluene solution was placed in a restricted, axially symmetric geometry composed of a spherical lens on a Si substrate (i.e., sphere-on-Si geometry), forming a capillary-held solution. The solvent evaporation led to the self-assembly of $\gamma-\mathrm{Fe}_{2} \mathrm{O}_{3}$ nanoparticles into hundreds of spatially well-ordered, gradient concentric 'coffee rings'. The rings were two nanoparticle layers high with a monolayer of nanoparticle thin film formed in between. The ring width ranged from submicron to a few microns. The center-to-center distance between adjacent rings was on the order of a few microns. The size and packing of $\gamma-\mathrm{Fe}_{2} \mathrm{O}_{3}$ nanoparticles, both within individual microscopic rings and in between, were dependent upon the preparation of the $\gamma-\mathrm{Fe}_{2} \mathrm{O}_{3}$ nanoparticle toluene solution.

S. Supplementary data are available from stacks.iop.org/JPhysCM/21/264014

(Some figures in this article are in colour only in the electronic version)
\end{abstract}

\section{Introduction}

Assemblies and controlled positioning of metallic, semiconducting, and magnetic nanoscale materials into well-ordered functional structures offer new opportunities for miniaturized electronic, optoelectronic, and magnetic devices $[1,2]$. Their collective properties depend on particle size, shape, and spatial arrangement of the nanomaterials. The latter can be produced by employing a variety of self-assembly techniques, including self-directed self-assembly [2], magnetic fieldinduced assembly [3], and electrostatic interaction-induced assembly [4]. Evaporative self-assembly of nonvolatile solutes (e.g., nanoparticles and polymers) from a sessile drop stands out as an extremely simple route to creating intriguing one- or two-dimensional structures [5-9]. In general, surface patterns produced by the far from equilibrium evaporation process include polygonal network structures [7, 10-12], fingering instabilities [9, 13], and stochastic multiple 'coffee rings' without controllable spacing and size $[5,14,15]$. In the latter context, maximum evaporative loss of solvent at the edge of a sessile drop promotes the jamming of nonvolatile solutes and creates a local roughness. The solutes dispersed in solution are then transported toward the edge and deposited at the

\footnotetext{
1 Author to whom any correspondence should be addressed.
}

contact line (i.e., 'stick'), i.e., forming a 'coffee ring'. The drop then undergoes a jerk (i.e., 'slip') to a new position, resulting in a new 'coffee ring'. The 'stick' and 'slip' motions of the contact line alternate as the solvent evaporates and ultimately lead to the formation of concentric "coffee rings'. However, randomly arranged multi-rings are generally yielded [14, 15]. Therefore, to produce intriguing, wellordered structures via evaporation, it is essential to control the evaporation flux, solution concentration, interfacial interaction between the solute and the substrate, etc.

Iron oxides (e.g., $\gamma-\mathrm{Fe}_{2} \mathrm{O}_{3}$ and $\mathrm{Fe}_{3} \mathrm{O}_{4}$ ), among the most attractive magnetic materials, have been extensively studied in recent years. Iron oxide nanoparticles are excellent candidates for use in studies of nanoscale magnetic properties [16, 17] and have been widely used in magnetic data storage, ferrofluid technology [18], energy storage, lubricants, drug delivery [19, 20], and magnetic resonance imaging (MRI) [21]. Herein, we report on a simple route to producing highly ordered concentric 'coffee rings' composed of magnetic $\gamma$ $\mathrm{Fe}_{2} \mathrm{O}_{3}$ nanoparticles over a large surface area by allowing a drop of $\gamma-\mathrm{Fe}_{2} \mathrm{O}_{3}$ nanoparticle toluene solution to evaporate in a sphere-on-Si geometry. The implementation of sphereon-Si geometry eliminated the temperature gradient and the associated convective flow, thus providing control of the 


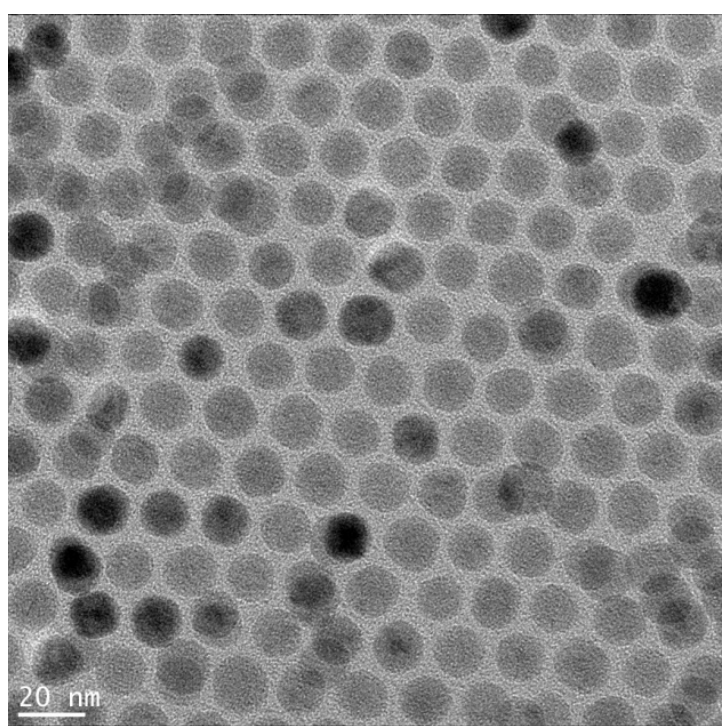

Figure 1. TEM image of $\gamma-\mathrm{Fe}_{2} \mathrm{O}_{3}$ nanoparticles, showing uniform size and shape of the nanoparticles. The diameter of the $\gamma-\mathrm{Fe}_{2} \mathrm{O}_{3}$ nanoparticle is $D=14 \mathrm{~nm}$. Scale bar $=20 \mathrm{~nm}$.

solution evaporation [1, 22-32]. The resulting concentric rings were two nanoparticle layers high, and submicron to a few microns in width. The center-to-center distance between adjacent rings was a few microns. This technique is remarkably controllable and easy to implement, opening up a new route for fabricating highly structured functional materials and devices in a fast, robust, and cost-effective manner.

\section{Experimental details}

\subsection{Synthesis and dispersion of $\gamma-\mathrm{Fe}_{2} \mathrm{O}_{3}$ nanoparticles}

Monodispersed $\gamma-\mathrm{Fe}_{2} \mathrm{O}_{3}$ nanoparticles were synthesized according to well-established procedures [33]. The synthesis is composed of two steps: synthesis of iron oleate complex followed by subsequent thermal decomposition of iron oleate. Briefly, a certain amount of iron chloride and sodium oleate (TCI chemicals) were dissolved in the mixture of DI water, ethanol and hexane at the volume ratio of 4:3:7 and heated to $70{ }^{\circ} \mathrm{C}$ for $4 \mathrm{~h}$. The upper organic phase was extracted using a separation funnel and washed with DI water three times. The iron oleate obtained was sealed and stored in a desiccator. $\gamma-\mathrm{Fe}_{2} \mathrm{O}_{3}$ nanoparticles were obtained by subsequent thermal decomposition of iron oleate in 1 -octadecene at $320^{\circ} \mathrm{C}$ for 30 min, using oleic acid as the surface capping ligand, for improving their solubility in organic solvents. After cooling to room temperature, the $\gamma-\mathrm{Fe}_{2} \mathrm{O}_{3}$ nanoparticles were purified by adding a large amount of ethanol and redissolving in toluene. The size of the $\gamma-\mathrm{Fe}_{2} \mathrm{O}_{3}$ nanoparticles synthesized was approximately $14 \mathrm{~nm}$ as determined by TEM (figure 1). The $\gamma-\mathrm{Fe}_{2} \mathrm{O}_{3}$ nanoparticle toluene solution at the concentration $c=0.06 \mathrm{mg} \mathrm{ml}^{-1}$ was purified either once with a $0.2 \mu \mathrm{m}$ hydrophobic PTFE membrane filter (denoted as normally filtered solution) or twice (i.e., with a $0.2 \mu \mathrm{m}$ PTFE membrane filter, followed by a glass microfiber filter (GF/C, with $42.5 \mathrm{~mm}$ circles from Whatman)) (denoted as

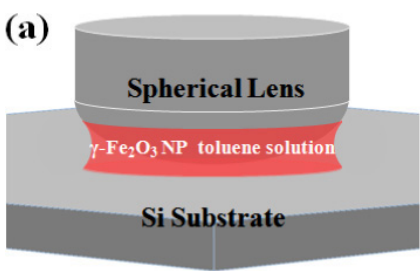

(b)

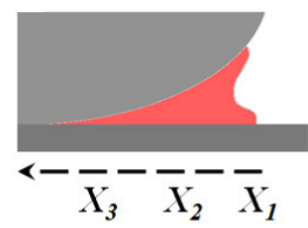

Figure 2. (a) Schematic three-dimensional illustration of a capillary-held toluene solution containing $\gamma-\mathrm{Fe}_{2} \mathrm{O}_{3}$ nanoparticles placed in a sphere-on-Si geometry. (b) A close-up of the right capillary edge in (a) (side view), where $X$ is the distance from the sphere and Si contact center. $X_{1}, X_{2}$, and $X_{3}$ refer to outermost, intermediate, and innermost regions, respectively.

intensively filtered solution). The intensity from the intensively filtered solution in the absorbance measurement was lower than that from the normally filtered solution (data not shown), suggesting that the concentration of intensively filtered solution was reduced as compared to that of the normally filtered solution.

\subsection{Evaporative self-assembly of $\gamma-\mathrm{Fe}_{2} \mathrm{O}_{3}$ nanoparticle rings in a sphere-on-Si geometry}

A drop of $\gamma-\mathrm{Fe}_{2} \mathrm{O}_{3}$ nanoparticle toluene solution (volume $V=18 \mu \mathrm{l}$; NPs) was loaded and trapped within a small gap between a spherical lens made of fused silica and a native silicon oxide-terminated silicon substrate due to the capillary force. To implement a sphere-on-Si geometry (figure 2(a)), an inchworm motor with a step motion of a few micrometers was used to place the upper sphere into contact with the lower stationary Si substrate. The experiment was performed inside a sealed transparent chamber, so that the evaporation rate was controlled and the temperature gradient was eliminated during the experiment. A capillary-held nanoparticle solution was formed with the evaporation rate being highest at the extremity (figure 2(b)). The diameter and radius of curvature of the sphere are $1 \mathrm{~cm}$ and $2 \mathrm{~cm}$, respectively. The evaporation took approximately $30 \mathrm{~min}$ to complete, after which the two surfaces were separated. Due to the curving effect of the upper sphere, only the surface structures formed on the Si substrate were evaluated.

\subsection{Characterization}

TEM imaging was performed using a JEOL 2100 scanning transmission electron microscope (STEM) operating at $200 \mathrm{kV}$. The surface structures produced on the $\mathrm{Si}$ substrate were characterized by optical microscopy (OM; Olympus BX51 in the reflection mode) and atomic force microscopy (AFM; Dimension 3100 scanning force microscope in the tapping mode (Digital Instruments)). BStap300 tips (Budget Sensors) with spring constants ranging from 20 to $75 \mathrm{~N} \mathrm{~m}^{-1}$ were used as scanning probes.

\section{Results and discussion}

Magnetic $\gamma-\mathrm{Fe}_{2} \mathrm{O}_{3}$ nanoparticles with $14 \mathrm{~nm}$ diameter were synthesized according to a reported procedure of nonhydrolytic 
thermal decomposition of iron oleate in high boiling point organic solvent $[3,33]$. To study the evaporative self-assembly of $\gamma-\mathrm{Fe}_{2} \mathrm{O}_{3}$ nanoparticles in the sphere-on-Si geometry, the $\gamma$ $\mathrm{Fe}_{2} \mathrm{O}_{3}$ nanoparticle toluene solution was loaded, and bridged the sphere and Si substrate, yielding a capillary-held solution (figure 2). The evaporation of toluene only occurred at the constrained capillary edge (figure 2(b)). As toluene evaporated, $\gamma-\mathrm{Fe}_{2} \mathrm{O}_{3}$ nanoparticles were transported from the solution to the capillary edge, conglomerated, and pinned the contact line (figure 3(a)), forming ringlike deposits. During the deposition of nanoparticles, the initial contact angle gradually decreased to a critical contact angle, at which the capillary force becomes larger than the pinning force, causing the contact line to jump to a new position, where it was arrested again and a new ring was thus produced [27]. The use of axially symmetric sphere-on-Si geometry was responsible for consecutive pinning (i.e., 'stick') and depinning (i.e., 'slip') cycles of the contact line in a controllable manner, leaving behind hundreds of concentric 'coffee rings' of $\gamma-\mathrm{Fe}_{2} \mathrm{O}_{3}$ nanoparticles (figure 3 ). The rings exhibited gradient features, representing the decrease in center-to-center distance between neighboring rings, $\lambda$ and the ring width, $w$, as the liquid capillary edge approached the sphere/Si contact center. This is because of the imbalance between the nonlinear capillary force, due to the curvature effect of the sphere, and the linear pinning force [27]. Two representative optical micrographs of highly ordered concentric nanoparticle rings were obtained from drying a droplet of normally filtered $\gamma-\mathrm{Fe}_{2} \mathrm{O}_{3}$ solution and intensively filtered solution in the sphere-on-Si geometry. Locally, they appeared as parallel periodic stripe patterns (figures 3(b) and (c)). The rings of high regularity and fidelity shown in figures 3(b)-(c) are in sharp contrast to the "coffee rings' that were produced from a sessile drop of $\gamma-\mathrm{Fe}_{2} \mathrm{O}_{3}$ nanoparticle toluene solution on the $\mathrm{Si}$ substrate, where rings were randomly deposited (figure S1 in supporting information (available at stacks.iop.org/JPhysCM/21/264014)).

AFM measurements were performed to examine the detailed surface morphologies of the $\gamma-\mathrm{Fe}_{2} \mathrm{O}_{3}$ rings and the packing of nanoparticles within the ring. Two-dimensional AFM height images obtained from normally filtered and intensively filtered solutions are shown in figures 4 and 5, respectively, with the concentric rings locally stripe looking. The ring width, $w$, was dependent on the time of pinning of the nanoparticles. Subsequent depinning events for the solution front determined the center-to-center distance between the adjacent rings, $\lambda$. For the intensively filtered nanoparticle solution, as the solution front moved toward the sphere/Si contact center, $\lambda$ and $w$ progressively decreased from $\lambda=$ $4.1 \mu \mathrm{m}$ and $w=1.4 \mu \mathrm{m}$ at $X_{1}=3050 \mu \mathrm{m}$ (outermost region) to $\lambda=3.7 \mu \mathrm{m}$ and $w=1.1 \mu \mathrm{m}$ at $X_{2}=2625 \mu \mathrm{m}$ (intermediate region) to $\lambda=3.4 \mu \mathrm{m}$ and $w=0.8 \mu \mathrm{m}$ at $X_{3}=2150 \mu \mathrm{m}$ (innermost region) (figure 5). With increased proximity to the sphere/Si contact center, rings with smaller $w$ formed, indicating a shorter pinning time of $\gamma-\mathrm{Fe}_{2} \mathrm{O}_{3}$ nanoparticles at the three-phase contact line. A smaller $w$ also implies a lesser volume loss of solvent during pinning [27]. As a result, this led to a shorter pull away of the contact line to a new position, thereby yielding a smaller $\lambda$ (figure 5) [27]. It is
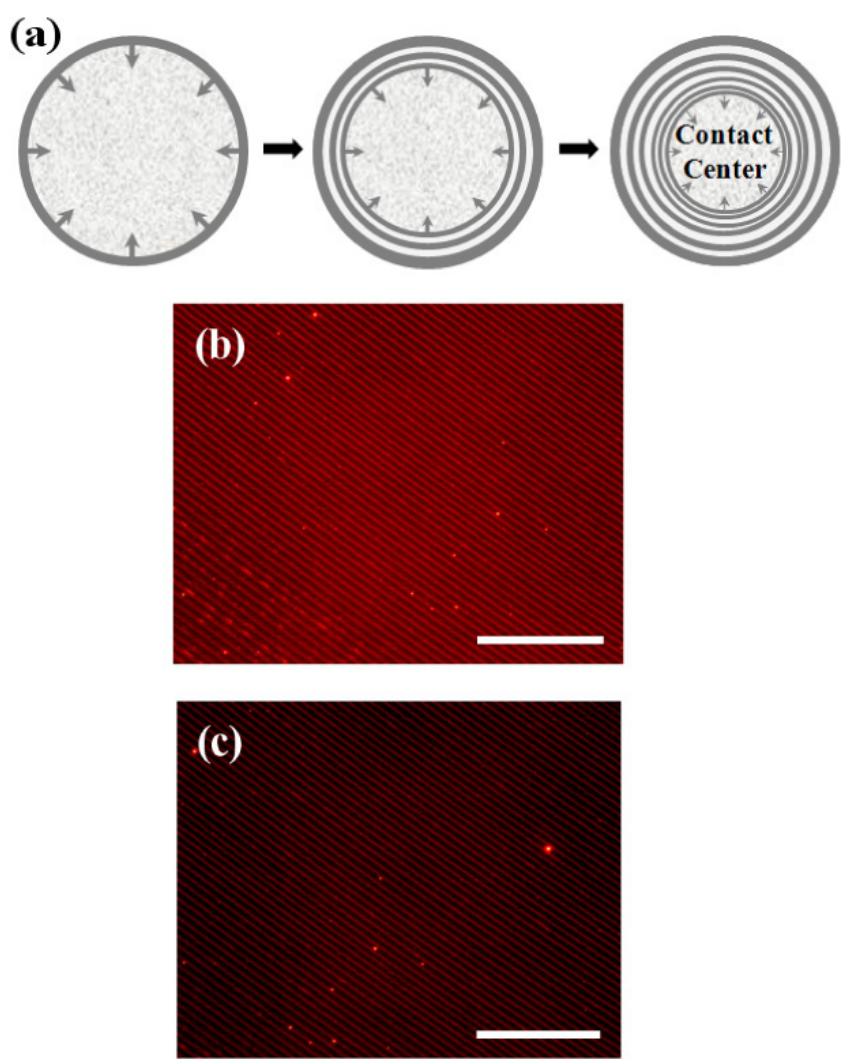

Figure 3. (a) Schematic drawings (top view) depicting the sequential formation of gradient concentric $\gamma-\mathrm{Fe}_{2} \mathrm{O}_{3}$ nanoparticle rings during the solvent evaporation in the sphere-on-Si geometry shown in figure 2. ((b), (c)) Optical micrographs of ordered $\gamma-\mathrm{Fe}_{2} \mathrm{O}_{3}$ nanoparticle patterns obtained from evaporative self-assembly of $\gamma-\mathrm{Fe}_{2} \mathrm{O}_{3}$ nanoparticle toluene solution. (b) The $\gamma-\mathrm{Fe}_{2} \mathrm{O}_{3}$ nanoparticle solution filtered with a $0.2 \mu \mathrm{m}$ PTFE membrane filter was used in the experiment (denoted as normally filtered solution). (c) The $\gamma-\mathrm{Fe}_{2} \mathrm{O}_{3}$ nanoparticle solution purified with $0.2 \mu \mathrm{m}$ PTFE membrane filter followed by a glass microfiber filter was employed (denoted as intensively filtered solution). Scale bar $=70 \mu \mathrm{m}$.

worth noting that when quantum dots were used as nonvolatile solutes, a transition from rings to wirelike structures was observed [29]. Such a transition was not seen in the case of $\gamma$ $\mathrm{Fe}_{2} \mathrm{O}_{3}$ nanoparticles. Micron (figures 5(a)-(b)) and submicron (figure 5(c)) width concentric $\gamma-\mathrm{Fe}_{2} \mathrm{O}_{3}$ nanoparticle rings were produced exclusively on the $\mathrm{Si}$ substrate. For the normally filtered nanoparticle solution, however, rings were only formed in the outermost region over a lateral distance of $500 \mu \mathrm{m}$, in which $\lambda=4.1 \mu \mathrm{m}$ and $w=1.1 \mu \mathrm{m}$ (figure 4); continuous film was then found in intermediate and innermost regions. A deposition of nanoparticles in the area between the rings is clearly evident (close-up; right panel in figure 4).

To measure the thickness of the rings as well as the deposits between the rings, AFM imaging was conducted on the sample after mechanically scratching to remove the nanoparticles off the Si substrate. The cross-section analysis on the scratched ring patterns revealed that the ring height, $h$, was almost constant over the entire deposited area (i.e., $h \simeq 28 \mathrm{~nm}$ at $X_{1}, X_{2}$ and $X_{3}$ from intensively filtered solution, and $h \simeq 28 \mathrm{~nm}$ from normally filtered solution), which is twice the diameter of the $\gamma-\mathrm{Fe}_{2} \mathrm{O}_{3}$ nanoparticles 


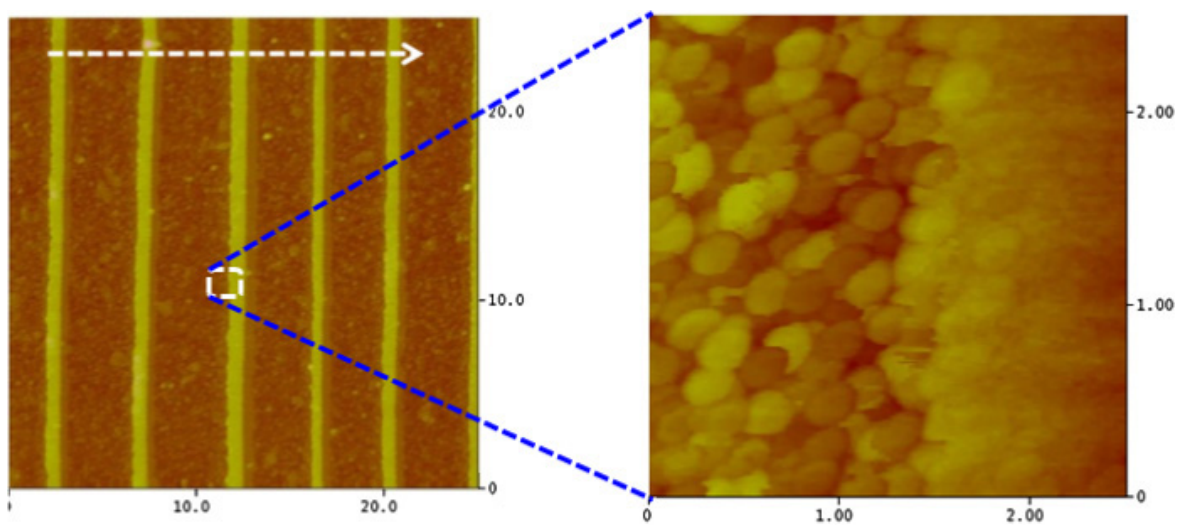

Figure 4. A representative AFM image of microscopic ring patterns at the outermost region, $X_{1}$, obtained from evaporative self-assembly of $\gamma-\mathrm{Fe}_{2} \mathrm{O}_{3}$ nanoparticle toluene solution (normally filtered solution). The scan sizes are $25 \times 25 \mu \mathrm{m}^{2}$ (left) and $2.5 \times 2.5 \mu \mathrm{m}^{2}$ (right; close-up of white square box in the left image), respectively. $Z$ scale $=100 \mathrm{~nm}$ and $40 \mathrm{~nm}$ for left and right images, respectively.
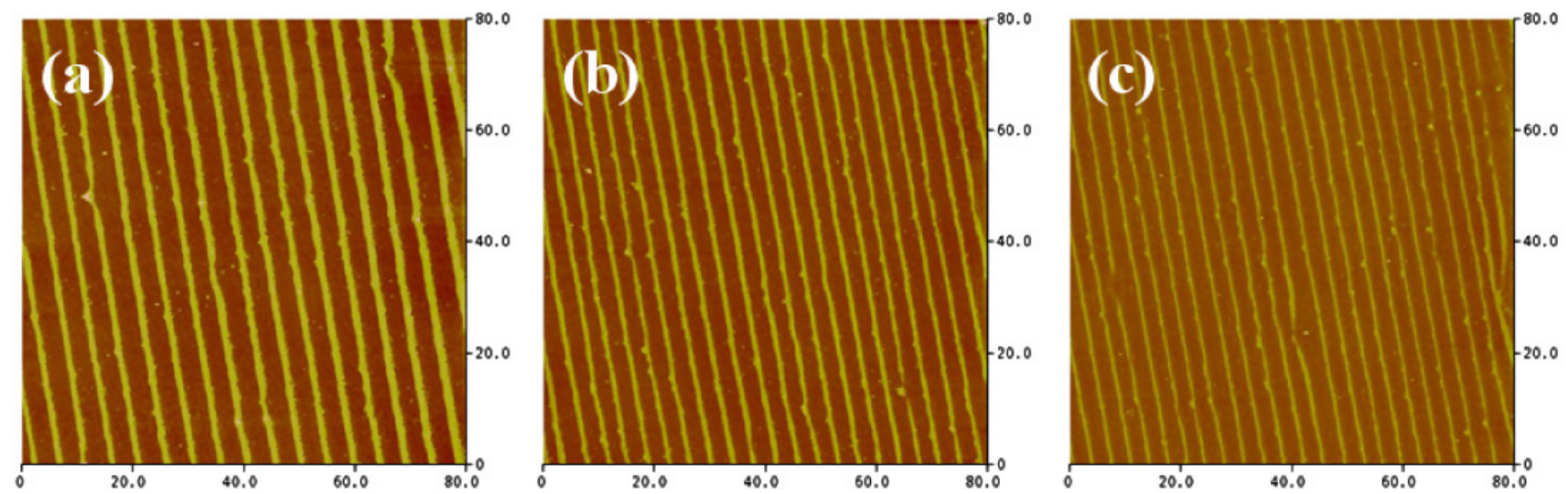

Figure 5. AFM height images of well-ordered $\gamma-\mathrm{Fe}_{2} \mathrm{O}_{3}$ nanoparticle rings formed in the different regions. The intensively filtered nanoparticle toluene solution was used in the experiment. (a) At the outermost region $\left(X_{1}\right)$, (b) at the intermediate region $\left(X_{2}\right)$, and (c) at the innermost region $\left(X_{3}\right)$. As the solution front moved toward the sphere/Si contact center due to the evaporative loss of toluene, gradient concentric rings emerged. Image size $=80 \times 80 \mu \mathrm{m}^{2}$.

$(D=14 \mathrm{~nm}$; figure 1), indicating the formation of a double-layer ring (figure S2 in supporting information (available at stacks.iop.org/JPhysCM/21/264014)). The constant $h$ measured likely reflected that, at the low solution concentration ( $c=0.06 \mathrm{mg} \mathrm{m}^{-1}$ ), only two layers of nanoparticles can be deposited to form a ring and no excessive nanoparticles were allowed to transport and deposit on the ring.

According to AFM images for the scratched samples, a monolayer of $\gamma-\mathrm{Fe}_{2} \mathrm{O}_{3}$ nanoparticles was formed in the area between the rings for both solutions. Comparison of the AFM images of $\gamma-\mathrm{Fe}_{2} \mathrm{O}_{3}$ nanoparticle surface morphologies obtained from different solutions revealed that the solution preparation method has a marked effect on the packing of nanoparticles within the rings and the monolayer films (figures 4 and 6). As shown in figure 4, the $\gamma-\mathrm{Fe}_{2} \mathrm{O}_{3}$ nanoparticle aggregates with average size of $200 \pm 10 \mathrm{~nm}$ were deposited from the normally filtered solution as is evidenced in the inter-ring region. By contrast, the aggregates were successfully removed by vigorously purifying the as-prepared solution with a $0.2 \mu \mathrm{m}$ PTFE membrane filter, followed by a glass microfiber filter (i.e., the intensively filtered solution; figure 6); much smaller $\gamma-\mathrm{Fe}_{2} \mathrm{O}_{3}$ nanoparticles were closely packed within the ring and in the inter-ring area.

The deposition of densely packed, monolayer nanoparticle thin film in the inter-ring area (close-up; right panel in figures 4 and 6) may be rationalized as follows. First, the passivation efficiency of $\gamma-\mathrm{Fe}_{2} \mathrm{O}_{3}$ nanoparticle with the organic ligand oleic acid may not be as good as in the case of tri- $n$-octylphosphine oxide (TOPO)-functionalized quantum dots [29]. The partial dissociation of oleic acid from the $\gamma-\mathrm{Fe}_{2} \mathrm{O}_{3}$ nanoparticle surface produced the hydrophilicity of nanoparticles, thereby facilitating the adsorption of nanoparticles to the hydrophilic, native silicon oxide-terminated Si substrate during the 'slip' process of the contact line. The detachment of oleic acid, if significant, may be responsible for the $\gamma-\mathrm{Fe}_{2} \mathrm{O}_{3}$ nanoparticle aggregates seen in figure 4. Second, due to the dissociation of oleic acid ligands and, thus, presenting of excess ligands in the solution, the rate of evaporation of toluene was retarded [34]. A slower evaporation rate may provide the nanoparticles with a chance to wet the hydrophilic surface (i.e., the interring area) during the 'slip' process and self-organize upon drying. 


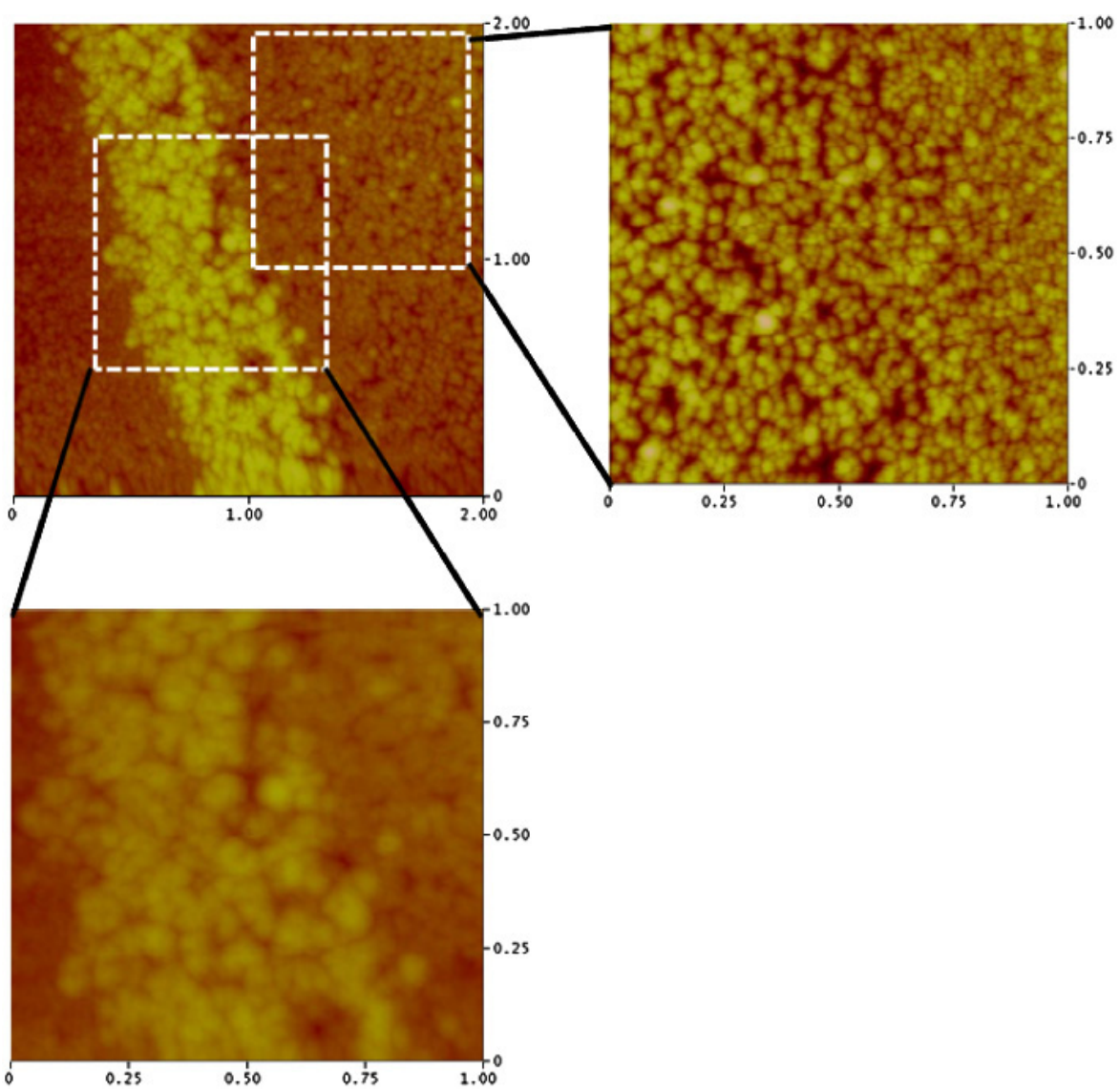

Figure 6. AFM height image, showing the packing of $\gamma-\mathrm{Fe}_{2} \mathrm{O}_{3}$ nanoparticles on the ring (forming a bilayer of $\gamma-\mathrm{Fe}_{2} \mathrm{O}_{3}$ nanoparticles) and in the inter-ring area (forming a monolayer thin film), obtained from evaporative self-assembly of intensively filtered nanoparticle toluene solution. Close-ups of the ring and the inter-ring area are shown in the lower panel and the right panel, respectively. Scan size $=2 \times 2 \mu \mathrm{m}^{2}$ in (a), and $1 \times 1 \mu \mathrm{m}^{2}$ in (b) and (c). $Z$ scale $=50 \mathrm{~nm}, 20 \mathrm{~nm}$, and $40 \mathrm{~nm}$ in (a), (b), and (c), respectively.

\section{Conclusions}

In summary, we have demonstrated that the use of axially symmetric sphere-on-Si geometry provided a regulation of the solvent evaporation, thereby promoting the self-assembly of magnetic nanoparticles into massively ordered concentric 'coffee rings'. The rings were two nanoparticle layers high, and submicron to a few microns in width. The distance between adjacent rings was on the order of a few microns. A monolayer of nanoparticle thin film was formed in between the rings. The size and packing of $\gamma-\mathrm{Fe}_{2} \mathrm{O}_{3}$ nanoparticles within an individual microscopic ring were dependent upon the preparation of $\gamma-\mathrm{Fe}_{2} \mathrm{O}_{3}$ nanoparticle toluene solution. These self-arranged, alternating double-layer (rings) and monolayer (thin films between the rings) magnetic nanoparticle patterns over large areas may offer potential applications in high density storage media, and bio-related devices such as biomolecular detectors, environmental monitors, and biological warfare agent sensing. We envisage the possibility of creating a family of surface patterns of different shapes and sizes via controlled, consecutive 'stick-slip' motions of the contact line, by replacing the upper spherical lens in the restricted geometry with different shapes [32], for example, a pyramidal lens or a cylindrical lens. The study of this is currently under way.

\section{Acknowledgments}

We gratefully acknowledge support from the National Science Foundation (NSF CAREER Award, CBET-0844084). We also thank Jun Xu for discussion and Qingze Zou for AFM usage. TEM characterization was carried out at the Microscopy and Nanoimaging Facility (MNIF) at Iowa State University.

\section{References}

[1] Hong S W, Jeong W, Ko H, Kessler M R, Tsukruk V and Lin Z Q 2008 Adv. Funct. Mater. 182114

[2] Lin Y, Boker A, He J, Sill K, Xiang H, Abetz C, Li X, Wang J, Emrick T, Long S, Wang Q, Balazs A and Russell T P 2005 Nature $\mathbf{4 3 4} 55$

[3] Ahniyaz A, Sakamoto Y and Bergstrom L 2007 Proc. Natl Acad. Sci. USA 10417570

[4] Kalsin A M, Fialkowski M, Paszewski M, Smoukov S K, Bishop K J M and Grzybowski B A 2006 Science 312420

[5] Deegan R D, Bakajin O, Dupont T F, Huber G, Nagel S R and Witten T A 1997 Nature 389827

[6] Gleiche M, Chi L F and Fuchs H 2000 Nature 403173

[7] Rabani E, Reichman D R, Geissler P L and Brus L E 2003 Nature 426271

[8] Bigioni T P, Lin X M, Nguyen T T, Corwin E I, Witten T A and Jaeger H M 2006 Nat. Mater. 5265

[9] Huang J, Kim F, Tao A R, Connor S and Yang P D 2005 Nat. Mater. 4896 
[10] Blunt M O, Martin C P, Ahola-Tuomi M, Pauliac-Vaujour E, Sharp P, Nativo P, Brust M and Moriarty P J 2007 Nat. Nanotechnol. 2167

[11] Martin C P, Blunt M O, Pauliac-Vaujour E, Stannard A and Moriarty P 2007 Phys. Rev. Lett. 99116103

[12] Moriarty P, Taylor M D R and Brust M 2002 Phys. Rev. Lett. 89248303

[13] Lyushnin A V, Golovin A A and Pismen L M 2002 Phys. Rev. E 65021602

[14] Adachi E, Dimitrov A S and Nagayama K 1995 Langmuir 111057

[15] Shmuylovich L, Shen A Q and Stone H A 2002 Langmuir 183441

[16] Ouyang M and Hiraoka H 1997 MRS Bull. 321099

[17] Romero-Perez M, Rincon J M, Oliver C, D'Ovidio C and Esparza D 2001 MRS Bull. 361513

[18] Rosensweig R E 1985 Ferrohydrodynamics (Cambridge: Cambridge University Press)

[19] Trewyn B G, Giri S, Slowing I I and Lin V S Y 2007 Chem. Commun. 3236-45

[20] Giri S, Trewyn B G, Stellmaker M P and Lin V S Y 2005 Angew. Chem. Int. Edn $\mathbf{4 4} 5038$

[21] Jeong U, Teng X, Wang Y, Yang H and Xia Y 2007 Adv. Mater. 1933
[22] Hong S W, Giri S, Lin V S Y and Lin Z Q 2006 Chem. Mater. 185164

[23] Hong S W, Xia J, Byun M, Zou Q and Lin Z Q 2007 Macromolecules 402831

[24] Hong S W, Xia J and Lin Z Q 2007 Adv. Mater. 191413

[25] Hong S W, Xu J and Lin Z Q 2006 Nano Lett. 62949

[26] Hong S W, Xu J, Xia J, Lin Z Q, Qiu F and Yang Y L 2005 Chem. Mater. 176223

[27] Xu J, Xia J, Hong S W, Lin Z Q, Qiu F and Yang Y L 2006 Phys. Rev. Lett. 96066104

[28] Wang J, Xia J, Hong S W, Qiu F, Yang Y and Lin Z Q 2007 Langmuir 237411

[29] Xu J, Xia J and Lin Z Q 2007 Angew. Chem. Int. Edn 461860

[30] Byun M, Hong S W, Zhu L and Lin Z Q 2008 Langmuir 243525

[31] Byun M, Hong S W, Qiu F, Zou Q and Lin Z Q 2008 Macromolecules 419312

[32] Hong S W, Byun M and Lin Z Q 2009 Angew. Chem. Int. Edn 48512

[33] Park J, An K, Hwang Y, Park J, Noh H, Kim J, Park J, Hwang N and Hyeon T 2004 Nat. Mater. 3891

[34] Lin X M, Jaeger H M, Sorensen C M and Klabunde K J 2001 J. Phys. Chem. B 1053353 